\title{
Frontières
}

\section{Le suicide dans les petites sociétés}

\section{Michel Tousignant}

Volume 12, numéro 1, automne 1999

Suicides, générations et culture

URI : https://id.erudit.org/iderudit/1074507ar

DOI : https://doi.org/10.7202/1074507ar

Aller au sommaire du numéro

\section{Éditeur(s)}

Université du Québec à Montréal

ISSN

1180-3479 (imprimé)

1916-0976 (numérique)

Découvrir la revue

\section{Citer cet article}

Tousignant, M. (1999). Le suicide dans les petites sociétés. Frontières, 12(1), 43-49. https://doi.org/10.7202/1074507ar

\section{Résumé de l'article}

Cette recension résume les écrits sur le suicide dans les petites sociétés, plus spécifiquement dans les Îles du Pacifique et la Nouvelle Guinée Papoue, et elle montre comment la théorie de Durkheim ne s'applique pas aux données contemporaines. Le suicide a existé longtemps avant l'acculturation radicale et sa relation au changement social est complexe. La contagion paraît jouer un rôle au niveau du village. Les statistiques officielles ne sont généralement pas valides lorsqu'on les comparent aux observations de terrain conduites avec une enquête approfondie sur les causes de décès. Les augmentations du taux de suicide rapportées récemment peuvent être partiellement attribuables à de meilleures méthodes de collecte des données. Le suicide dans les petites sociétés n'est pas associé à un processus d'aliénation sociale comme dans les sociétés urbaines et la présence de maladies psychiatriques y est moins évidente. Dans ces environnements, la conduite suicidaire est plus souvent caractérisée par un processus de réparation, et dont le but implicite est souvent d'atteindre une dignité personnelle ou familiale plutôt que le simple fait de vouloir se venger.
Ce document est protégé par la loi sur le droit d'auteur. L'utilisation des services d’Érudit (y compris la reproduction) est assujettie à sa politique d'utilisation que vous pouvez consulter en ligne.

https://apropos.erudit.org/fr/usagers/politique-dutilisation/ 


\section{Résumé}

Cette recension résume les écrits sur le suicide dans les petites sociétés, plus spécifiquement dans les îles du Pacifique et la Nouvelle Guinée Papoue, et elle montre comment la théorie de Durkheim ne s'applique pas aux données contemporaines. Le suicide a existé longtemps avant l'acculturation radicale et sa relation au changement social est complexe. La contagion paraît jouer un rôle au niveau du village. Les statistiques officielles ne sont généralement pas valides lorsqu'on les comparent aux observations de terrain conduites avec une enquête approfondie sur les causes de décès. Les augmentations du taux de suicide rapportées récemment peuvent être partiellement attribuables à de meilleures méthodes de collecte des données. Le suicide dans les petites sociétés n'est pas associé à un processus d'aliénation sociale comme dans les sociétés urbaines et la présence de maladies psychiatriques y est moins évidente. Dans ces environnements, la conduite suicidaire est plus souvent caractérisée par un processus de réparation, et dont le but implicite est souvent d'atteindre une dignité personnelle ou familiale plutôt que le simple fait de vouloir se venger.

Mots clés: suicide - anthropologie - culture - épidémiologie - Asie - Pacifique

\section{Abstract}

Suicide in small scale societies is not associated with a process of social alienation as in urbanized societies and the presence of psychiatric illness is less evident. Suicide in these settings is more characterized by a process of reparation, and its implicit goal is often to regain personal or family dignity rather than a simple act of revenge. This review summarizes reports coming from various cultural areas, especially the Pacific islands and Papua New Guinea, and shows how Durkheim's theory does not fit contemporary data. The other findings are the following: suicide has long existed before radical acculturation and its relationship to social change is complex; contagion seems to play a role at the village level; suicide is not always present when its main risk factors are observed; official statistics are usually not valid when a field study make a thorough enquiry of causes of death and recent increases of suicide may be partially due to better data collection.

Key words: suicide - anthropology - culture - epidemiology - Asia - Pacific

\section{Le suicide dans les petites sociétés*}

\author{
Michel Tousignant, \\ professeur au Département de psychologie, UQAM \\ Laboratoire de recherche en écologie humaine \\ et sociale (LAREHS).
}

Le suicide dans les petites sociétés nous offre une perspective unique pour améliorer notre compréhension du suicide. La recherche dans ces milieux repose presque uniquement sur la méthode anthropologique qui fournit une information détaillée en provenance de la famille et des amis. Le compte rendu typique décrit les événements déclencheurs, les réactions suite au suicide, l'interprétation sociale et religieuse, et les changements historiques récemment vécus au sein de la communauté. D'autre part, le partage de conditions de vie similaires dans ces petites sociétés est associé à des configurations similaires entourant le suicide, autant à l'intérieur de ces cultures qu'entre elles, comme dans le phénomène du suicide de vengeance aussi appelé suicide de Samson. D'un autre côté, la prévalence élevée de problèmes psychiatriques ou le fait de ne pas être marié n'est pas aussi étroitement associée au suicide dans ces sociétés comme cela peut l'être dans les milieux occidentaux ou métropolitains.

L'univers a subi des changements sociaux radicaux depuis la fin de la Seconde Guerre mondiale. L'éducation, le tourisme, l'économie basée sur la monnaie, la globalisation des communications, la baisse de l'âge médian des populations, la fin des guerres tribales et la nucléarisation de la famille sont les éléments essentiels de la transformation radicale de la culture, de la famille et du soi. Plusieurs sociétés ont été témoins de plus d'innovations au cours de cette période d'une génération qu'au cours des deux derniers millénaires.

Durkheim (1897) a émis une théorie selon laquelle le suicide dans le contexte européen de son époque était le produit d'une absence de normes et de la désintégration de la solidarité sociale. Il a utilisé les termes de suicide anomique et de suicide égoïste pour rendre compte de cette réalité. Afin d'expliquer le suicide dans les territoires colonisés éloignés, Durkheim a émis l'idée qu'une pression sociale trop forte mène au suicide et il a proposé la catégorie de suicide altruiste. Les données recensées ici ne correspondent pas toujours au modèle du suicide altruiste parce que la plupart de ces sociétés ont vécu un changement social radical et qu'elles ne sont pas aussi homogènes qu'autrefois. Mais, elles ne correspondent pas également à l'image des milieux anomiques des grandes cités où le suicide a tendance à se concentrer. Il y a effectivement un besoin de mettre à jour la classification de Durkheim et de se mettre à la recherche de nouveaux outils conceptuels.

Personne ne mettra en cause la vision selon laquelle le suicide est un acte extrême de détachement, le départ volontaire et radical du monde des êtres humains vivants. Dans les cultures métropolitaines, les individus qui commettent un suicide viennent en quelque sorte confirmer une mort déjà acquise sur le plan social. Des sentiments de solitude et de désespoir sont souvent caractéristiques de ceux qui meurent par suicide au moins parmi la population qui est sous soins psychiatriques (Ahrens et Linden, 1996). Les personnes décédées par suicide ont souvent été 
victimes de formes variées d'exclusion au sein de leur famille d'origine et, par la suite, ont souvent été rejetées par leur partenaire marital ou ont opéré une espèce de retraite dans l'alcoolisme et d'autres expressions de désordres mentaux. Leur mort marque la fin d'un sentiment profond d'aliénation sociale.

Ce vocabulaire est moins approprié pour les modes de vie traditionnelle articulés autour d'un rythme très dense d'échanges interpersonnels. Le suicide dans ces milieux peut être une forme de discours, une tentative désespérée de réparer les dommages dans le tissu social. L'expression suicide par vengeance ou suicide de Samson et autres synonymes ont été utilisés en référence aux formes spécifiques de suicide dans ce contexte. Le personnage biblique de Samson, (Livre des Juges 16:28) s'est comporté un peu comme un commando suicide entraînant dans la mort un très grand nombre de Philistins ainsi que luimême dans le but de venger la perte de sa vision.

Nous avançons l'idée que le suicide dans les petites sociétés peut être conçu comme un acte de réparation du lien entre le décédé et ses survivants. Un acte de réparation de l'intégrité de sa famille ou de sa propre réputation qui est généralement coextensive à celle de sa famille. Il y a certainement un élément agressif dans la plupart de ces suicides appelés suicide de vengeance. Mais dans ces sociétés holistiques, le suicide est davantage une façon de reconstituer une forme quelconque d'intégrité perdue qu'une fuite dans le monde des ténèbres.

Cette recension sera limitée aux populations résidant à la périphérie du monde industrialisé. Les groupes aborigènes de l'Australie et du Canada (Kirmayer, 1994) ne sont pas couverts. La structure du texte est faite en fonction des aires culturelles et géographiques.

\section{ÎLES DU PACIFIQUE}

Lîle de Tobi est un petit territoire isolé dans le sud Pacifique. La population a été dramatiquement réduite à seulement 60 membres au cours des récentes années suite à l'immigration dans le chef-lieu de Palau (Black, 1985). À l'époque de l'étude de Black, en 1972, l'économie reposait essentiellement sur la culture du taro et la monnaie était rarement impliquée dans les transactions. On était en mesure de répertorier autant que cinq suicides pendant une période de quelques années seulement. L'un de ces hommes décédés se remettait d'un amour non réciproque et un autre n'avait pas obtenu l'autorisation parentale de marier sa bien-aimée. Dans un autre exemple, deux jeunes amoureux, frustrés de la même manière, se sont enfuis en haute mer dans un petit canot et sont certainement morts malgré des rumeurs qui auraient atteint les Philippines à plusieurs centaines de milles au large, rumeur selon laquelle ils auraient eu douze enfants. Enfin, un homme s'est donné la mort en cessant de se nourrir, tout honteux parce qu'aucune femme ne pouvait lui procurer sa ration de taro. Ce geste a été perçu comme absurde parce que l'homme aurait pu s'acheter du riz à la place.

Les tentatives de suicide répétées d'Alfredo, observées en cours de terrain, ont procuré un matériel unique pour analyser le processus du suicide dans cette communauté. Cet homme divorcé avait la charge de plusieurs enfants, était tombé en amour avec une jeune fille et il l'avait en effet retirée du bassin limité des filles mariables. Cet événement a créé une tension sociale, mais la rumeur n'avait jamais atteint le niveau d'un outrage public avant que la mère de la fille ne fasse remontrance publiquement à Alfredo, criant à qui voulait l'entendre qu'il était un cochon qui se comportait envers sa propre fille comme si elle était sa propriété privée. Alfredo était grandement angoissé par cette apostrophe. Il disparut dans la forêt et fut trouvé un peu plus tard sur la branche d'un arbre, jouant avec une corde. À plusieurs occasions, par la suite, il a menacé de se tuer mais les villageois s'en sont moins préoccupés, croyant qu'il se remettait progressivement de l'humiliation subie.

Dans une société comme celle de Tobi, fondée sur une grande confiance entre les concitoyens pour leur support matériel et émotionnel, le pouvoir de la honte peut annihiler une personne. Dans ce contexte, le suicide est attribué à une honte suffisamment forte pour produire un état dissocié de conscience. La personne se transforme en un esprit, se retire de la vie sociale, et peut éventuellement tuer ses voisins ou commettre un suicide. Lorsque l'amour et l'approbation des voisins sont si fondamentaux pour l'identité, le rejet ne laisse aucune autre façon de fuir que l'immigration.

La Samoa occidentale est un autre groupe qui a été durement frappé par le suicide avec un taux de 23 pour 100000 pour 1981-1983, ce qui est encore malgré tout en dessous du taux de 48 pour la Micronésie (MacPherson et MacPherson, 1987). Les jeunes hommes entre 15 et 29 ans sont principalement respon- sables de cette augmentation. Le taux de suicide a doublé en 1976 et a continué d'augmenter lentement par la suite. Les statistiques ont raté plusieurs cas parce qu'il n'y avait pas d'obligation légale d'établir la cause de décès. Les familles étaient aussi soupçonnées de tenter de cacher un suicide parce qu'il signifiait un échec à résoudre un conflit et un manque de "leadership» au sein de la famille. De même, les suicides se produisant dans des villages éloignés, dans le maquis ou en haute mer sont difficiles à confirmer. Enfin, la population locale croit en toute bonne foi que quelques suicides sont causés par les esprits (aitu) et sont rapportés comme des accidents.

Le suicide relié à la honte a été une forme traditionnelle de suicide en Samoa occidentale. La mort y est vue comme une réparation pour le tort occasionné par le décédé qui menaçait de causer un problème à son groupe. La famille était alors épargnée d'une poursuite en cours et d'un procès public. Un dicton local soulignait que la mort était préférable à la honte malgré le tabou religieux entourant le suicide. MacPherson et MacPherson, (1987) attribue le suicide à un processus de retrait, un concept mis de l'avant par Merton pour rendre compte d'une situation d'attente élevée et de faible opportunité pour atteindre des objectifs culturels, ce qui forçait l'individu à avoir recours à ses propres ressources personnelles. Une manifestation publique de rage accompagne souvent ces suicides; quelques individus ont consommé des herbicides en présence de leurs proches afin de provoquer une réaction de remords.

Jusqu'au milieu des années 1970, l'immigration en Samoa américaine ou en Nouvelle-Zélande offrait une possibilité pour les nouveaux arrivants sur le marché du travail, mais ces frontières se sont éventuellement refermées. À la même époque l'augmentation de l'espérance de vie encourageait une structure de pouvoir gérontocratique et bloquait les voies à la mobilité sociale. Ceci était reflété par le fait que le suicide frappait surtout la portion éduquée de la jeune population.

Suite à une augmentation des suicides parmi les jeunes gens de l'île de Truk (une partie du territoire en tutelle des îles du Pacifique) une enquête approfondie a été conduite par Hezel (1984). Le suicide n'était pas complètement inconnu avant cette époque: une recherche des archives du 19e siècle rapportait des agrégats de suicide et de tentatives de suicide parmi les étudiants et les familles du personnel scolaire pen- 
dant la période coloniale. En effet, les suicides étaient si fréquents qu'une «institutrice a jugé nécessaire de former une équipe de garçons pour couper la corde des victimes en cas d'urgence à venir» (p. 193). Des contagions de suicide sont aussi rapportées pour cette période dans les îles avoisinantes de Guam, Ponape et Gilbert.

Hezel a recueilli des informations en profondeur sur 129 suicides entre 1971 et 1983 dans cette population de 40000 habitants. Il a interviewé des amis et des survivants de la personne décédée par suicide. Le taux qu'il a obtenu, beaucoup plus élevé que dans les archives publiques, s'élevait à 30 pour 100000 pour une période de trente ans. Quatrevingt-seize cas sont suffisamment détaillés pour les classifier dans une typologie qui inclut la colère $(n=74)$, la honte $(n=15)$, et la psychose $(n=7)$. Comme dans la Somoa occidentale, le taux a été multiplié par six autour de l'année 1975, partiellement dû à des méthodes de recueil des données plus fiables.

Le suicide dans l'île de Truk est caractérisé par un ratio masculin-féminin de 12:1 et une moyenne d'âge de seulement 19 ans. Il y a eu autant que onze enfants de moins de quatorze ans qui se sont suicidés durant la période de l'étude, un nombre égal au total des suicides pour ce groupe d'âge dans la plupart des grands pays occidentaux. Les hauts taux de Micronésie se retrouvent à la périphérie des centres urbains, avec des niveaux intermédiaires d'acculturation. Pas plus de $10 \%$ des cas démontrent des signes évidents d'alcoolisme ou d'une autre maladie psychiatrique.

Une enquête sur les tentatives de suicide dans une population de 1500 personnes de plus de dix ans a produit un nombre total de 100 tentatives de suicide (Hezel, 1984). Toutefois, deux informateurs clés, un prêtre local et un intervenant en santé mentale, étaient d'opinion qu'au moins $50 \%$ de la population adulte avait fait une tentative de suicide véritable, et que, éventuellement, la population avait cessé de faire attention à ce phénomène.

Un tel taux de conduites suicidaires parmi la jeune population dans une aire géographique si réduite amène immédiatement à penser à la possibilité de séries de suicide ou de suicide par imitation. Dans une analyse de 400 cas en Micronésie (Rubinstein, 1987) plus de $20 \%$ des personnes décédées par suicide avaient un ami proche ou un parent, parfois résidant dans la même maison, qui sont morts également par suicide. On a compté cinq suicides dans un court laps de temps au sein d'un petit village alors que, des cas de suicide étaient plutôt exceptionnels ailleurs sur la même île. Quelquefois la preuve de la contagion était convaincante. Un jeune homme s'est pendu sur la tombe de son ami qui venait tout juste de décéder par suicide. Un autre garçon a utilisé le même arbre et la même corde que son ami pour le suivre dans la mort cinq jours plus tard. Les croyances culturelles offrent des explications religieuses pour ces épidémies qui deviennent une source de grande angoisse. Un scénario appelé le dilemme des amoureux rend compte de plus de vingt suicides. Dans le premier récit de cette série, un jeune homme est tombé amoureux de deux filles en même temps et il a eu un enfant de chacune d'elle. Un autre garçon plus tard a contribué à romancer cette forme de suicide en écrivant sur le mur de son école, "Adieu (des deux amis de cœur). J'ai apprécié d'être avec chacune de vous!»

Le suicide chez ces jeunes était souvent déclenché par un conflit d'apparence triviale avec la famille. Par exemple, un enfant chantait trop fort ou on refusait de lui acheter une nouvelle chemise (Hezel, 1984). Ces suicides sont impulsifs et ne sont pas associés à une dépression ou à une maladie mentale grave. Le lien affectif avec les membres de la famille est généralement fort et l'adolescent semblait préférer mourir plutôt que d'altérer le lien avec sa famille suite à des frustrations répétées et à l'accumulation d'une colère réprimée. Il y avait souvent une histoire familiale de conflits chroniques et graves. En agissant dans le contexte d'une insulte de peu de conséquences, le jeune exprimait une rage sans implication de vengeance, ce qui aurait pu être le cas si le suicide avait été précédé d'un comportement parental abusif. Les membres de la famille n'avaient pas l'impression généralement que la mort constituait une vengeance et ils manifestaient des signes authentiques de deuil aux funérailles. Hezel souligne que le geste suicidaire avait la coloration d'un acte de se prendre en pitié, un sentiment connu sous le nom de amwunumwun, mélange de retrait et d'abaissement de soi pour exprimer comment une personne se sent atteinte. Plusieurs enfants ont même manifesté un souci à propos de l'opinion que leur famille aurait d'eux après leur mort. Il y avait également deux cas où le suicide avait été accompli dans le but de forcer une réconciliation entre d'autres membres de la famille.
La moitié des suicides ont été accomplie sur l'influence d'une intoxication alcoolique élevée, mais l'alcool agissait comme un catalyseur plutôt que comme une cause selon l'auteur (Hezel, 1987). L'alcool était généralement consommé par des jeunes gens pour ventiler leur ressentiment à l'égard des aînés. À long terme, cette habitude de consommation augmentait l'agressivité plutôt que de la réduire. Cependant, une psychopathologie grave, la délinquance ou l'incompétence sociale étaient rarement présentes dans ces suicides.

La forme plus traditionnelle de suicide de honte dans l'île de Truk avait habituellement comme origine une mauvaise conduite menaçant la réputation de la famille. Des liaisons amoureuses illicites avec un premier cousin, avec la femme d'un frère ou avec le fiancé d'une fille étaient les types d'événements qui provoquaient la colère pour les suicides de personnes très jeunes. Les garçons impliqués réagissaient principalement à la peur d'être frappés et à la ou peur d'être battus ou d'être réprimandés par leur parent.

Même si ces suicides se sont produits au cours d'une période de modernisation intense, il n'y a pas de liens apparents avec l'acculturation. Ceux qui se sont suicidés étaient généralement fidèles à la tradition. La culture était souvent incapable de procurer des solutions aux nouveaux défis de la vie. Un changement fondamental amené par la modernisation dans l'île de Turk et en Micronésie et le passage d'une structure matrilinéaire organisée autour de l'autorité des frères de la mère à la nucléarisation de la famille (Hezel, 1987; Rubinstein, 1983, 1987). L'économie monétaire a brisé le réseau de distribution de nourriture au sein du clan et a donné une autorité plus forte au père biologique sur ses enfants. Mais cette génération de pères n'avait pas de modèle de rôle sur la façon d'élever les enfants. Les oncles étaient moins prêts à interférer dans les affaires de famille et le réseau de sécurité d'un parentage multiple se trouvait perdu. Les enfants ont commencé à s'enorgueillir de leur souffrance et à en faire d'une façon de manipuler les parents et la conduite suicidaire est devenue un outil formidable de chantage. La question posée est de savoir si le taux élevé de suicide se limitera à cette période transitionnelle dans les rôles familiaux ou si elle s'étendra à la prochaine génération si les modèles de parentage demeurent confus.

La plupart de ces récits de la région $\mathrm{du}$ Pacifique ne correspondent pas 
facilement aux catégories de Durkheim de suicide anomique, égoïste ou altruiste. L'acte suicidaire constitue souvent l'application d'une norme culturelle dans le contexte d'un changement social et de valeurs en conflit et il n'est pas réellement anomique. Il n'y a pas de solution pour la réparation sociale et le maintien de la dignité. La mort est préférée à la honte pour conserver ou réparer la bonne réputation de la famille. Ailleurs, les jeunes gens ne veulent pas déranger la paix de la famille (et celle de la communauté) en exprimant leur colère et en défiant la structure d'autorité. L'apparente absence de psychopathologie grave est probablement un signe que la personne suicidaire ne réagit pas à un désespoir personnel mais plutôt à une contradiction sociale.

\section{PAPOUE NOUVELLE-GUINÉE}

Les conflits maritaux sont à la source de la plupart des suicides féminins rapportés en Papoue Nouvelle-Guinée. Parmi les Maring, les mères tuent souvent leurs enfants en même temps en signe de vengeance pour priver leur mari de nouvelles alliances de parenté (Healy, 1979). Les Counts (1987, Counts et Counts, 1991) ont décrit quatre suicides féminins parmi les Kalai dont trois d'entre eux ont pris place dans le contexte d'une violence domestique. Les femmes détruisaient généralement leur propriété avant de se suicider et elles s'habillaient avec leurs plus beaux vêtements pour cette occasion. Les époux soupçonnaient que leur femme avait eu une relation extra-maritale ou qu'une preuve d'adultère était à l'origine de ces conflits maritaux. La famille de l'épouse pouvait réagir à ce suicide. Ainsi, un mari a été assassiné par sa belle-famille et un autre est mort de maladie peu après avoir subi un mauvais sort.

Les travaux de Johnson (1981) parmi les Gainj montrent que la violence domestique a augmenté suite à une tentative européenne de mettre fin aux guerres intertribales Le fait d'assumer une autorité sur leurs épouses est devenue la seule façon pour ces guerriers retraités d'affirmer leur masculinité. Ayant probablement joui de plus d'indépendance lorsque leur mari était aux combats, ces femmes abusées préféraient la mort à une soumission honteuse. Elles croyaient également qu'elles pouvaient se venger sur leur mari dans l'après-vie. Quelques-unes de ces femmes se sont pendues dans des lieux publics pour humilier leur partenaire. Les maris étaient souvent ridiculisés pour ne pas avoir pu contrôler leur femme et ils avaient à retourner la dot. Ces cas de suicide procuraient aussi aux autres femmes une espèce de levier dans la négociation de leurs conflits maritaux.

Une enquête sur les BiminKuskusmin (Poole, 1985) a fourni de plus amples preuves que les hauts taux de suicide dans les quelques-unes des petites sociétés précédaient un contact étroit avec la civilisation industrialisée et qu'ils étaient présents au cours d'une période où le cannibalisme n'avait pas été complètement éradiqué. Autant que $10 \%$ des décès sur les dernières six générations ont pu être attribuées au suicide. Durant les deux années de la recherche, au début des années 1970, 30 suicides ont rendu compte de $57 \%$ de tous les décès. De plus, il y a eu deux tentatives de suicide de la part d'enfants de cinq et sept ans. Pour chaque suicide féminin, il $\mathrm{y}$ avait six femmes qui faisaient une menace sérieuse. Les hommes qui commettaient un suicide étaient principalement âgés entre 23 et 34 ans c'est-à-dire, l'âge de faire la guerre, et ils souffraient apparemment d'un ressentiment à l'égard des exigences très élevées des obligations de la guerre. Les femmes étaient considérablement insatisfaites de leur condition de vie parce que leurs hommes passaient leur temps à se tuer entre eux et qu'elles-mêmes devaient manger les victimes. Des tentatives de condamner le suicide de façon stricte n'ont pas concouru d'aucune façon à atténuer le phénomène.

On peut voir que le suicide parmi les femmes Gainj est une façon d'obtenir une réparation pour leur famille d'origine lorsque le cadeau qu'elle représentait était en quelque sorte gaspillé par leur mari et par leur belle-famille. D'autre part, les suicides parmi les hommes de Bimin-Kuskusmin insatisfaits avec l'état de guerre et les femmes insatisfaites avec leur condition de vie en temps de guerre sont plus difficiles à intégrer à un modèle de réparation. Ici, le suicide peut être interprété comme une forme de protestation par les faibles et plusieurs suicides peuvent être vus comme une tentative pour compenser pour les conditions de vie profondément insatisfaisantes.

\section{INDONÉSIE}

Hollan (1990) a réitéré l'opinion selon laquelle la théorie de l'anomie ne correspondait pas à la plupart des suicides parmi la tribu Toraja des hautes terres de l'Indonésie. Ce groupe est très acculturé suite à l'éducation et à un style de vie urbain. La vie quotidienne cependant est encore soumise aux règles d'échanges généralisées, imbriquées dans des processus amorcés il y a plusieurs générations.
L'auteur souligne que les Toraja jouissent d'un faible taux de suicide mais que le type de suicides observés ressemble à ce qu'on retrouve en Micronésie. Il propose que le comportement suicidaire létal et non-létal, spécifiquement parmi la jeune population, est provoqué par un sentiment frustré d'avoir le droit à une éducation supérieure et à consommer des biens matériels parce que leurs parents ont préféré dépenser leur argent pour des rituels. Un profond sentiment de frustration combiné à une incapacité de ces jeunes gens d'exprimer leur colère à l'égard de leur parent mène à un type de suicide que Hollan appelle indignité. Il explique la dynamique culturelle du geste en soulignant que les requêtes des adolescents étaient en général culturellement légitimes, quoique différentes de celles de l'ancienne génération. Son argument manque d'un appui empirique et la preuve est principalement tirée de cas de menace de suicide qui se sont montrés efficaces pour contrer le contrôle parental, surtout dans le choix d'un partenaire marital.

Bali est un exemple intéressant d'une petite population distincte religieusement de ses voisins et avec un taux de suicide bas malgré l'introduction du tourisme international et d'un changement social rapide. À l'occasion d'une recension sur la culture et la maladie mentale à Bali, Suryani (1995) mentionne très peu de choses à propos du suicide sinon une référence au fait que les tentatives de suicide se produisent généralement dans le contexte d'un état de transe. Les facteurs associés au suicide tels: l'abus parental, la consommation de drogue, la délinquance juvénile, la dépression et même la schizophrénie et la personnalité multiple sont rapportés comme rares dans cette culture.

\section{ASIE DU SUD}

Daniel (1989) commente le suicide dans une communauté de nouveaux Tamouls du Nord-Centre du Sri Lanka qui sont arrivés de l'Inde au cours du dernier siècle et qui ont immigré récemment de la région de Vavuniya après avoir été déplacés des plantations de thé. Le suicide dans cette région a atteint un sommet de 78 pour 100000 dans les années 1970 et il y avait lieu de croire que le taux était aussi très élevé parmi les nouveaux Tamouls. Les nouveaux Tamouls comprennent seulement $17 \%$ de la population de 1978 de Vavuniya, mais leur arrivée a coïncidé avec une augmentation par quatre du taux de suicide. Les statistiques deviennent non fiables après 1978 à cause de l'étendue 
de la guerre civile, mais la communauté locale croit également que le taux a véritablement décru.

Le suicide parmi les nouveaux Tamouls semble correspondre à une situation d'anomie à cause du caractère dépressif des cas suite au déracinement de la population de son territoire, (Tanimai tosam) qui se traduit littéralement comme «maladie de la solitude» a été identifiée comme la cause proximale de plusieurs suicides chez les nouveaux Tamouls. Donc, utilisant une logique complexe, basée sur la théorie sémiotique de Pierce, Daniel suggère que la diminution apparente du suicide à la fin des années 1970 n'était pas tant attribuable à moins d'anomie ou à un changement dans les conditions de vie qu'à une réorientation des émotions négatives à l'égard des manifestations de violence armées raisonnées contre l'armée cingalaise.

\section{MAORIS}

Les Maoris de la Nouvelle-Zélande sont connus depuis la colonisation pour avoir une tolérance élevée à l'égard du suicide (Skegg, Cox, et Broughton, 1995). Il y a une falaise réputée comme lieu de suicide et le port de Whangaroa dériverait son nom du mot suicide. La mort de la veuve aussi connue comme sati en Inde et le suicide de honte sont rapportés dans les mémoires de Dieffenbach en 1843. Écrivant 20 ans plus tard, Manning croit que le suicide est un fait presque quotidien parmi les Maoris. Les récits oraux mentionnent également le suicide. L'un de ces récits rapporte que Te Aohuruhuru, la jeune épouse d'une grande beauté, avait été exposée nue à d'autres hommes par son mari pendant son sommeil (Sachdev, 1990). Pour se venger, la femme a sauté d'une falaise dans la mer face au bateau de son mari.

$\mathrm{Au}$ cours de la dernière décennie (1982-1991) le taux de suicide parmi les Maoris a été moins élevé que la moyenne nationale des non-Maoris, phénomène principalement attribuable à un très faible taux parmi les femmes et les Maoris plus âgés (Skegg et al., 1995). Dans la catégorie des 15-24 ans, le taux des Maoris est similaire au taux du même groupe d'âge de NouvelleZélande qui occupe un haut rang sur le plan international. Le haut taux parmi les jeunes Maoris ne peut pas être interprété comme la conséquence de l'acculturation. Les facteurs de risque et les scénarios de suicide diffèrent significativement entre les groupes culturels. Entre 1980 - 1988, 23 \% des suicides masculins de Maoris dans cette catégorie d'âge ont pris place dans le contexte d'une prison ou de garde policière. Les jeunes Maoris emprisonnés réagissent souvent avec le whakama, une réaction de honte caractérisée par un sentiment d'infériorité et une perte de prestige (Sachdev, 1990). Leur sens d'appartenance est un caractère encore fortement présent dans leur identité et une condamnation pénale menace de les ostraciser aux yeux de leur communauté. Les jeunes Maoris possèdent également un taux de décrochage scolaire et de chômage deux fois plus élevé que les non-Maoris. Leur taux d'admission à l'hôpital pour dépendance à l'alcool et à la drogue est aussi très élevé de même que leur taux de blessures infligées malgré le fait que le taux de suicide est légèrement plus bas.

De nouveau, nous avons encore ici une situation où la honte et la répression de la réputation familiale jouent probablement un rôle. Cependant, nous avons un problème d'aliénation sociale d'une population marginalisée dans un pays grandement industrialisé.

\section{AUTRES COMMUNAUTÉS ISOLÉES}

\section{GROENLAND}

Les taux élevés de suicide observés récemment dans les nations aborigènes acculturées (Kirmayer, 1994) ont aussi été observés parmi les Inuits $\mathrm{du}$ Groenland. Le suicide, rare au cours de l'époque coloniale, a récemment subi un bond, atteignant le taux 0,5\% par année, probablement le taux le plus haut sur le plan international (Lynge, 1995). Il y a de grandes différences entre les aires géographiques et il n'y a aucune association directe avec le taux de l'acculturation (Grove et Lynge, 1979). L'abus d'alcool, les taux élevés d'homicide et de conflits familiaux sont aussi présents dans cette communauté.

\section{CARAÏBE}

Le taux de suicide de la Jamaïque est très bas à 1,5 pour 100000 en $1975-1976$ et à 0,5 pour 100000 en 1988 - un taux inférieur même dans le contexte des Caraïbes (Wedenoja, 1995). La Jamaïque possède une prévalence élevée d'un certain nombre de facteurs habituellement associés au suicide tels l'usage répandu de la marijuana, du cannabis, des taux élevés de famille sans père $(70 \%)$, de familles d'accueil à l'intérieur de la famille étendue $(63 \%)$ et de conduite antisociale. Le système matriarcal d'éducation des enfants peut jouer un rôle comme filet de sécurité en encourageant l'enfant à s'attacher à plusieurs figures adultes telles la grand-mère et la sœur de la mère. Ceci peut protéger contre des sentiments de rejet et d'abandon ou amoindrir la nécessité d'idéaliser la mère, ce qui réduit ainsi le risque de se sentir trahi lorsque des frustrations se produisent.

\section{AFRIQUE}

Seulement un rapport récent d'Afrique s'applique à une petite société, celle des Sara Nar qui vivent dans une région marécageuse du Centre-Sud du Chad (Brown, 1981). La population de 20000 habitants est distribuée dans des villages d'environ 450 personnes. À l'époque de l'étude de terrain, le mode de vie était encore très traditionnel, fondé sur la culture du millet et du sorgho sans la présence d'une pression démographique sur les terres. Une enquête approfondie a révélé 12 suicides incluant deux sœurs adolescentes sur une période de trois ans entre 1971 et 1973, ce qui produit un taux de 20 pour 100 000, très élevé pour l'Afrique rurale.

Les Sara Nar attribuent le suicide soit à une colère produite par le deuil ou soit à des remarques désobligeantes. Aucun cas cependant n'a été relié au deuil, mais il y avait plusieurs suicides provoqués par des insultes. Le scénario typique était celui d'une personne qui tentait de prouver son innocence en protestant contre ceux qui avaient élevé une charge contre lui. Plusieurs cas étaient reliés à la perte de statut. Quelques femmes avaient perdu leur enfant mâle, un homme était sexuellement impuissant et trois hommes n'arrivaient pas à contrôler leur épouse.

Le fait que le suicide soit plus élevé parmi des adultes mariés que parmi les adolescents est probablement un signe de continuité de vie traditionnelle. L'auteur propose que les suicides sont provoqués par un excès d'exigences culturelles associées au statut mais avec peu de moyens pour les atteindre. Aucun cas ne correspondait à l'hypothèse de l'anomie ou de l'aliénation sociale.

\section{AMÉRIQUE LATINE}

Il y a eu très peu de recherches sur le suicide parmi les groupes isolés de l'Amérique latine. Une publication en provenance des Guarana du Nord $d u$ Paraguay a trouvé un ratio élevé de suicide féminin-masculin. L'agent déclencheur était généralement l'abus psychologique ou physique de la part du mari quand celui-ci n'aimait pas la façon dont sa femme accomplissait ses travaux domestiques. Il y avait aussi des exemples de vengeance de la part de la famille de l'épouse. Les femmes utilisaient souvent les menaces de suicide 
pour acquérir une forme quelconque de contrôle domestique.

À l'occasion d'une enquête épidémiologique couvrant 16 villages Quichua des hautes terres du Bolivar en Équateur entre 1960 et 1980 , le suicide est d'environ de 9 par 100000 dans la population âgée de 18 ans et plus (Tousignant et Chela, 1990-1991). Trois des suicides rapportés étaient le fait de jeunes hommes qui se sentaient incompris de la part de leur parent. Les récits étaient similaires à ceux rapportés dans l'île de Truk et étaient déclenchés par des conflits mineurs. Deux frères se sont tués après que leur père eut refusé de les prendre pour un voyage. Au moins un suicide féminin et quelques conduites non létales sont attribués à des conflits avec la belle-mère et à l'absence de protection patrilocale de la part du mari. Le mariage patrilocal est souvent une source importante d'angoisse quand la jeune femme ne peut pas compter sur sa propre famille pour l'appuyer.

Harner (1972) a étudié les Jivaro de l'Amazonie et les quelques cas décrits dans sa monographie suffisent à pouvoir extrapoler un haut taux pour cette population à faible densité. Les Jivaro considèrent une conduite similaire aux Kamikazes comme un suicide. Lorsque les guerriers sont frustrés de la vie, ils se portent volontaires pour être au front lors de raids homicidaires avec peu de chances de survie. Une telle décision est considérée comme le reflet de la perte de l'âme. Les femmes se suicident généralement par pendaison. Elles sont habituellement victimes d'abus de la part de leur mari ou elles sont trouvées coupables d'adultère, un crime qui est punissable de mutilation corporelle.

\section{LES TERRITOIRES EUROPÉENS}

La Réunion est un territoire français dans l'océan Indien. La population compte un peu plus d'un demi million d'habitants en 1985 , soit le double de celle de 1960. Elle inclut des noirs, des blancs créoles et des indiens de la côte du Malabar. L'âge moyen est un peu audessus de 20 ans et l'âge moyen du suicide est de 39 ans (Reverzy, Duval, Vanelven et Cazalis de Fondouce, 1987). Le taux de suicide a triplé entre 1961 et 1983 pour atteindre un taux de 18 pour 100 000. L'amélioration des méthodes d'enregistrement sont partiellement responsables de cette augmentation. Le taux de chômage de $37 \%$ dans cette société principalement agricole peut être relié au haut taux de suicide chez les jeunes hommes. Plus de $35 \%$ des cas d'urgence psychiatriques sont le fait d'une tentative récente de suicide et le taux annuel de traitements pour tentatives de suicide dans le territoire des deux principaux hôpitaux est de 0,5\%; $55 \%$ des tentatives sont le fait de personnes de moins de 25 ans et $25 \%$ de personnes de moins de 20 ans avec un ratio féminin-masculin de 3:2.

Les îles de Canari comprennent une population isolée d'origine européenne et qui compte 1,5 million d'habitants. La tendance des taux de suicide a suivi les taux de l'Espagne et de l'Europe (Rodriguez-Pulido, Sierra, Doreste, Gracia et Gonzalez-Rivera, 1991; Rodriguez-Pulido, Sierra, Gracia, Doreste, Delgado et Gonzalez-Rivera, 1991). Il y a eu une augmentation graduelle de 6,8 à 8,1 pour 100000 entre 1977 et 1983 et le suicide est corrélé avec l'âge. Ces taux sont fondés sur l'application des critères ICD-9 et ils sont deux fois plus élevés que ceux des archives officielles. La raison principale de cet écart est que le premier certificat temporaire de décès spécifie simplement la nature $\mathrm{du}$ processus biologique menant à la mort, (par exemple un œdème pulmonaire aigu ou un traumatisme cranio-encéphalite). Il y a un examen judiciaire très long dans le cas des morts violentes et la décision d'un suicide n'est souvent pas communiquée au registre civil.

\section{DISCUSSION ET CONCLUSION}

Cette recension sur le suicide dans les petites sociétés montre comment les sociétés autochtones ont été durement frappées par le phénomène du suicide durant la période de changements sociaux rapides qui a suivi la Seconde Guerre mondiale. Ceci est vrai pour de nombreux groupes particulièrement en Micronésie, en Papoue NouvelleGuinée, au Groenland et parmi les Maoris de la Nouvelle-Zélande. Bien qu'il n'y ait pas de relation directe avec l'acculturation, les changements de style de vie ont certainement joué un rôle majeur. Le suicide a souvent été présent avant l'époque de la colonisation et certaines formes de suicide étaient intégrées aux processus sociaux traditionnels comme dans le cas de la perte de statut ou de l'abus conjugal.

La théorie de Durkheim, fondée principalement sur les concepts du suicide anomique et égoïste, a été l'objet de nombreuses critiques comme ayant été incapable de rendre compte des formes de suicide rapportées de ces sociétés. Les observateurs n'ont pas été particulièrement frappés par l'anomie ou l'aliénation sociale ou encore par une haute fréquence des problèmes psychiatriques. Le suicide était conçu comme un lien dans la chaîne du processus de restructuration des normes plutôt que comme la conséquence d'un vide social. Les normes continuent de survivre, mais les moyens pour les atteindre ont été renouvelés. Par exemple, les guerriers doivent trouver d'autres moyens de prouver leur masculinité, les parents d'autres stratégies pour manifester leur amour et leurs soins. Nous avons proposé que la réparation $\mathrm{du}$ statut individuel ou familial peut être un meilleur moyen d'interpréter une certaine partie de ces suicides plutôt que de simplement faire appel au concept de suicide par vengeance ou par indignité. Cela ne veut pas dire qu'un processus d'aliénation sociale n'est pas présent dans certains contextes de déracinement ou de changements sociaux extrêmes.

Lester (1995) a voulu donner un nouvel élan à la théorie du suicide de Narroll (1970) baptisée «thwarting disoriention» théorie qui se voulait un essai pour comprendre le suicide dans les petites sociétés. Les travaux de Narroll n'ont été que très peu cités par les suicidologues et les anthropologues, peut être parce que le concept manque de clarté. Exprimé en de simples termes, ce concept réfère à la frustration de satisfaction à laquelle on aurait droit, ce qui mènerait à une souffrance non voulue. Narroll a opérationnalisé ce concept à travers un très large éventail d'indicateurs depuis l'abus physique des femmes jusqu'à la fréquence des accusations d'ensorcellerie. Bien que sa méthode ait pu mener à de nombreuses associations positives, à partir des «Human Relation Area Files», la méthode a aussi reçu de nombreuses critiques (LeVine, 1973), certaines partagées par Narroll lui-même (1970). Cette théorie dérivée de la théorie de MillerDollard sur la frustration-agression tente probablement de couvrir trop de phénomènes pour rendre compte de la configuration actuelle du suicide.

Une caractéristique importante $d u$ suicide dans les petites sociétés par comparaison avec les sociétés occidentales est que les cas recensés ne se retrouvent pas à la périphérie de ces sociétés. Bien que nous nécessiterions davantage de données sur le statut clinique des personnes qui se suicident dans ces populations, il n'apparaît pas que le phénomène soit étroitement associé à la maladie mentale. Le suicide est moins souvent la conséquence d'un lent processus de désintégration sociale qui mène à un désespoir final qu'une stratégie radicale soit de repentir, soit de vengeance ou 
soit de réconciliation. Une approche à privilégier pour l'avenir serait d'analyser les sociétés qui possèdent un grand nombre de facteurs généralement associés au suicide sans que cette société présente un niveau élevé de suicide. La Jamaïque en est un bon exemple. Est-ce que dans ce dernier pays, la consommation de rhum et de cannabis dans des rencontres sociales ritualisées aiderait à prévenir quelques-unes des conséquences néfastes de ces produits? Les nombreuses séparations d'avec la mère ou le père ont probablement des conséquences pour la santé mentale, mais la présence d'un réseau de sécurité avec des figures d'attachement représentées par des figures parentales peuvent être instrumentales pour éviter le désespoir et le suicide. La protection pourrait provenir d'un appui émotionnel direct ou d'une absence d'idéalisation des parents biologiques. Par comparaison, dans la région du Pacifique, la nucléarisation de la famille empêche la régulation des conflits par la parenté.

Quelques observations dans les petites sociétés amènent à penser que le suicide par contagion est un phénomène réel. Le fait que plusieurs personnes décédées par suicide fassent partie d'un même réseau rend plausible l'hypothèse de la contagion. La religion peut aussi jouer un rôle en se faisant promotrice de la croyance que les esprits des êtres décédés viennent séduire les survivants ou encore que les candidats au suicide peuvent s'attendre à une meilleure vie après la mort et à la possibilité de jouir d'une vengeance. La principale question qui mérite réponse est de savoir pourquoi le suicide peut s'étendre à un petit village lorsque les villages qui l'environnent en sont épargnés?

Enfin, il est important de noter que les auteurs de plusieurs de ces études s'en rapportent au propre taux qu'ils ont calculé plutôt qu'aux archives officielles. Nous devrions être prudents à propos des taux qui ne sont pas validés indépendamment par des critères internationaux. Dans la plupart des cas, cela exige un travail de terrain. Ce problème est actuel non seulement dans les territoires éloignés, là où l'administration a peu d'influence mais aussi dans les populations administrées par des bureaucraties européennes. Des réserves s'imposent quant à la comparaison des taux entre plusieurs de ces communautés que nous avons recensées ici, surtout lorsque les critères et les procédures de classification de décès ne sont pas comparables.

\section{Références}

AHRENS et LINDEN, 1996.

BLACK, P.W. (1985). Ghosts, Gossip, and Suicide: Meaning and Action in Tobian Folk Psychology. In WHITE, G.M. and KIRKPATRICK, J.: PERSON, Self, and Experience, p. 245-300. Berkeley and Los Angeles, U. of California Press.

BROWN, E.P. (1981). The Ultimate Withdrawal: Suicide among the Sara Nar, Archives Européennes de Sociologie, 22, 199-228.

COUNTS, D.A. (1987). Female Suicide and Wife Abuse: A Cross-Cultural Perspective. Suicide and Life-Threatening Behavior, 17, 194-204.

COUNTS, D.A. \& COUNTS, D.R. (1991). Loss and Anger: Death and the Expression of Grief in Kaliai. In D.A. COUNTS \&. D.R. COUNTS (Eds.), Coping with the Final Tragedy: Cultural Variation in Dying and Grieving, (p. 191212). Amityville (New York): Baywood. DANIEL, E.V. (1989). The Semeiosis of Suicide in Sri Lanka. In B. Lee \& G. Urban (Eds.), Semiotics, Self, and Society, (p. 69-100). Berlin: Mouton de Gruyter. GROVE, O. \& LYNGE, I. (1979). Suicide and Attempted Suicide in Greenland. A Controlled Study in Nuuk. Acta Psychiatrica Scandinavica, 60, 375-391. HARNER, M. (1972). The Jivaro: People of the Sacred Water-falls. New York: Doubleday.

HEZEL, F.X. (1984). The Cultural Patterns in Trukese Suicide. Ethnology, 23, 193-206.

HEZEL, F.X. ((1987). In Search of the Social Roots of Mental Pathology in Micronesia. In A.B. ROBILLARD \& A.J. MARSELLA (Eds.), Contemporary Issues in Mental Health Research in the Pacific Islands, (p.12-31). Honolulu, HI: University of Hawaii Press.

HOLLAN, D. (1990). Indignant Suicide in the Pacific: An Example from the Toraja Highlands of Indonesia. Culture, Medicine, and Psychiatry, 14, 365-380.

JOHNSON, P.L. (1981). When Dying is Better than Living: Female Suicide among the Gainj of Papua New Guinea. Ethnology, 20, 325-334.

KIRMAYER, L. J. (1994). Suicide among Canadian Aboriginal Peoples. Transcultural Psychiatric Research Review, 31, 358.

LEVINE, R.A. (1973). Culture, Behavior, and Personality. Chicago, Aldine.

LYNGE, I. (1995). Modernization and Mental Illness in Greenland. In I. Al-Issa (Ed.). Handbook of Culture and Mental Illness: An International Perspective, (p. 269-280). Madison, Connecticut: International Universities Press.

MACPHERSON, C. \& MACPHERSON, L. (1987). Towards an Explanation of Recent Trends in Suicide in Western Samoa. Man, 22, 305-330.

NARROLL, R. (1970). What we Learned from Cross-Cultural Surveys? American Anthropologist, 72, 1227,1288.

POOLE, F.P. (1985). Among the Boughs of the Hanging tree: Male Suicide among the Bimin-Kuskusmin of Papua New Guinea. In F.X. HEZEL, D.H. RUBENSTEIN \&
REVERZY, J.F., DUVAL, G., VANELVEN, G. \& CAZALIS de FONDOUCE, G. (1987). Suicide et insularité: la réalité réunionaise [Suicide and Insularity: The Reality of La Réunion]. Psychologie Médicale, 19, 623-628.

RODRIGUEZ-PULIDO, F., SIERRA, A., DORESTE, J., GRACIA, R.\& J.L. GONZALEZ- DORESTE, J. (1991). Suicide in the Canary Islands: Standardized Epidemiological Study by Age, Sex, and Marital Status. Social Psychiatry and Psychiatric Epidemiology, 27, 69-74.

RODRIGUEZ-PULIDO, F., SIERRA, A., GRACIA, R, DORESTE, J, DELGADO S. \& GONZALEZ-RIVERA, J.L. (1991) Suicide in the Canary Islands, 1977-1983. Acta Psychiatrica Scandinavica, 84, 520523.

RUBINSTEIN, D.H. (1987). Cultural Patterns and Contagion: Epidemic Suicide among Micronesian Youth. In F.X. HEZEL, D.H. RUBENSTEIN \& G.H. WHITE (Eds.), Culture, Youth and Suicide in the Pacific: Papers from the East-West Center Conference, (p. 127148). Honolulu, HI: East-West Center.

RUBINSTEIN, D.H.. (1983). Epidemic Suicide among Micronesian Adolescents. Social Science and Medicine, 10, 657-665. SACHDEV, P.S. (1990). Whakama: Culturally Determined Behaviour in the New Zealand Maori. Psychological Medicine, 20, 433-444.

SKEGG, K., COX, B. \& BROUGHTON, J. (1995). Suicide among New Zealand Maori: Is History Repeating Itself? Acta Psychiatrica Scandinavica, 92, 543-459.

SURYANI, L.K. (1995). Cultural Factors, Religious Beliefs, and Mental Illness in Bali: Indonesia. In I. AL-ISSA (Ed.). Handbook of Culture and Mental Illness: An International Perspective, (p. 203214). Madison, Connecticut: International Universities Press.

TOUSIGNANT, M. \& CHELA, T. (19901991). Suicide in the Third World: The Case of Ecuador. Omega, Journal of Death and Dying, 23, 191-198.

WEDENOJA, W. (1995). Social and Cultural Psychiatry of Jamaicans, at Home and Abroad. In I. AL-ISSA (Ed.). Handbook of Culture and Mental Illness: An International Perspective, (p. 215230). Madison, Connecticut: International Universities Press.

WHITE, G.H. (Eds.), Culture, Youth and Suicide in the Pacific: Papers from the East-West Center Conference, (p. 152181). Honolulu, HI: East-West Center.

\section{Note}

Sous le titre «Suicide in Small-Scale Societies», la version originale de ce texte a été publiée dans un numéro spécial, «Culture and Suicide», de la revue Transcultural Psychiatry, Université McGill, vol. 35, no 2, juin 1998 p. 291-306. La revue nous a aimablement accordé l'autorisation d'en publier la version française. La traduction est de l'auteur. 